\title{
Kinds of Tropes without Kinds
}

[draft of the article published in dialectica, the definitive version of the article available at Wiley.com] https://onlinelibrary.wiley.com/doi/10.1111/1746-8361.12256

\author{
Markku Keinänen \\ Tampere University \\ Jani Hakkarainen \\ Tampere University \\ Antti Keskinen \\ Tampere University
}

In this article, we propose a new trope nominalist conception of determinate and determinable kinds of quantitative tropes. The conception is developed as follows. First, we formulate a new account of tropes falling under the same determinates and determinables in terms of internal relations of proportion and order. Our account is a considerable improvement on the current standard account (Campbell 1990; Maurin 2002; Simons 2003) because it does not rely on primitive internal relations of exact similarity or quantitative distance. The internal relations of proportion and order hold because the related tropes exist; no kinds of tropes need be assumed here. Second, we argue that there are only pluralities of tropes in relations of proportion and order. The tropes mutually connected by the relations of proportion and order form a special type of plurality, tropes belonging to the same kind. Unlike the recent nominalist accounts, we do not identify kinds of tropes with any additional entities (e.g. sets) or abstractions from entities (e.g. pluralities of similar tropes).

\section{Introduction}

Tropes are thin particular natures, such as an -e charge in some specific location. According to trope bundle theories, tropes form the only fundamental category. Trope bundle theories have two basic tenets. One is to construe objects and other entities by means of aggregates of tropes that fulfil some specific conditions. Here, an important aspiration is to provide a trope theoretic 
analysis of monadic inherence, or how tropes function as particular properties of objects (e.g. the redness of a rose or the -e charge of an electron). The other basic tenet is to account for objects having the same properties (e.g. two electrons both being -e charged) in nominalist terms, without postulating real generality such as property universals.

In trope theories, dealing with the second issue reduces to accounting for tropes being of the same kind. Consider two electrons having an -e charge. Assume that these determinate charges are tropes. One can ask: why are they both -e charge tropes? Why are these two tropes and a third trope - say, a particular e charge - all charge tropes - that is, of the same determinable kind? This article provides a new trope nominalist answer to these two questions regarding the division of tropes into determinate and determinable kinds. Scientific discourse involves general talk about determinable and determinate basic quantities (e.g. mass, charge, spin, and their specific values) in the formulation of the laws of nature. We will argue that the trope nominalist can account for this general talk without the postulation of general entities even considered as construed from tropes (e.g. sets of tropes).

In section 2.1, we outline the standard conception of tropes as thin particular natures, such as an -e charge or redness in some specific location. Moreover, we argue for a sparse theory of tropes, according to which the best candidates for tropes are basic physical quantities like determinate electric charges, rest masses, and colour charges. By contrast, we present empirically motivated reasons to reject certain of the most paradigmatic quality tropes, namely colours and shapes. Section 2.2 presents the standard conception of tropes belonging to the same kind. The basic idea of the standard conception is that tropes of the same determinate kind (e.g. redness tropes) resemble exactly, whereas tropes falling under the same determinable (colour tropes) resemble inexactly to some degree.

We present a new conception of the internal relations between quantity tropes falling under a determinate/determinable in sections 2.3-2.5, which replaces the standard 
conception. According to it, the tropes fall under the same determinate/determinable because they are mutually related by the internal relation(s) of proportion and order. For instance, all e charge tropes are mutually related by the relation of $1: 1$ proportion and are equal in order. Due to being determined by quantity tropes, the relations of proportion and order remain invariant in all changes of the conventional unit of any quantity. In section 2.6 , we argue that our conception of internal relations between tropes is superior to the standard conception in its explanatory power. We will construct a disjunctive argument containing two horns: either there are only quantity tropes or there are both quantity and quality tropes. With regard to the first horn, we will argue that, in contrast to the standard conception, our conception is able to eliminate the irreducible reference to determinables in the specification of quantitative distances (inexact resemblances) between quantity tropes. Therefore, it is superior to the standard view in this particular case. The standard view still has the prima facie advantage of being able to specify the internal relations between quality tropes of a determinate kind, namely exact resemblances. Nevertheless, we argue that quality tropes (if there are such) falling under a determinate can be similar and differ from each other in different respects. Thus, exact resemblances between quality tropes must be relativized to determinables. Since the respect relative similarities are not good candidates for internal relations, the apparent advantage is frustrated.

In section 3, we argue against currently popular views of kinds of tropes that attempt to identify kinds with abstract entities. Moreover, we take up A.R.J. Fisher's reductive identificationist view, which attempts to identify kinds of tropes with tropes and is a recent development of Williams's Moderate Realism. Although being interestingly economical, we argue that the reductive identificationist view fails. Finally, we defend an eliminativist view: there are no kinds or shared properties. We are capable of general talk by using expressions 
applying to pluralities of tropes standing in the relations of proportion and order. However, there is no good reason to introduce kinds of tropes as individual entities.

\section{Tropes of the same kind}

\subsection{Tropes}

Tropes are concrete (i.e. spatio-temporal or, at least, temporal) particulars. ${ }^{1}$ Trope theorists (i.e. trope-bundle theorists) assign to tropes the following standard category features of fundamental concrete particulars. First, in addition to being particular, tropes are categorially simple: either tropes are simple or all of their proper parts are tropes. Objects (and all particulars other than tropes) are individuals formed by the aggregates of tropes fulfilling some specific conditions. Second, tropes themselves are considered countable individuals having certain identity conditions (individuality) and countability (being one) as their further category features. Third, according to most trope theorists, tropes have determinate identity conditions independent of the identity of any other entity; they are identity-independent existents.

In addition to having specific category features, tropes are entities identified with a thin nature: a trope determines a single feature of the object possessing the trope (cf. Campbell 1990, 59-60; Simons 2003, sec. 6). We can label the view according to which every trope $t$ is identified with a determinate thin particular nature standard trope nominalism. ${ }^{2}$ In this

\footnotetext{
${ }^{1}$ Some trope theorists (such as Williams 1953a, 7-8; Campbell 1990, 53-6, and Denkel 1996) allow for the possibility of non-spatial (e.g. purely temporal) tropes. Both Williams (1953a) and Campbell $(1981,1990)$ call tropes "abstract particulars" because tropes occur as co-located with other tropes. Nevertheless, since it is (in their view) a contingent fact about every trope that it is co-located with other tropes (Williams 1953b, 179-80; Campbell 1981), Campbell's earlier conception of abstractness can be interpreted as epistemic: an entity is abstract if it comes before the mind by an act of abstraction, i.e. "by concentrating attention on some, but not all, of what is presented" (Campbell 1981, 477-8).

${ }^{2}$ The advocates of standard trope nominalism include Williams (1953a, 1953b), Campbell (1981, 1990), Maurin (2002), Simons (2003), and Giberman (2014). By contrast, Ehring (2011) claims that the nature of a trope must be determined extrinsically, by its belonging to a primitive natural class.
} 
framework, tropes are thin particular natures corresponding to intrinsic properties of objects. For instance, an -e trope determining the electric charge of an electron is the -e charge of that particular micro-particle. Since objects are capable of having distinct monadic properties, tropes can exist spatio-temporally co-located with each other. ${ }^{3}$ In standard trope nominalism, nature is a primitive concept or term, which is elucidated by examples. Nature might itself be considered quantitative (like e charge) or qualitative (a determinate shape) (cf. Fisher 2018, sec. 1). In this article, we adopt the standard trope nominalist conception of tropes as thin particular natures. We also assume that there is an intuitive distinction between quantities and qualities, which is sufficient for present purposes.

The favourite examples of tropes among metaphysicians are specific qualities, like the determinate redness of a rose. By contrast, according to a scientifically informed “empirical conception of property" (Balashov 1999, 257), the best examples of monadic particular properties are the tropes of basic physical quantities such as the tropes determining the intrinsic properties of elementary fermions (cf. Morganti 2009). For instance, a down-quark has (perhaps) a determinate -e/3 charge trope, a determinate rest mass trope, and a determinate spin quantum number trope among its proper parts. They determine certain causal powers of that micro-particle, which it has in some (approximate) location (Keinänen 2011, sec.4)

We take it to be the safest assumption in epistemic terms that there are concrete entities in causal interaction with us. Moreover, by correcting our prima facie beliefs, science - and especially physics - offers us the most reliable access to the intrinsic properties of concrete entities. The fundamental properties possessed by concrete entities are basic physical quantities (e.g. electric charges, spin quantum numbers, and quark colour charges), which are involved in the fundamental laws of nature, such as Coulomb's law (cf. Ellis 2001, 115;

\footnotetext{
${ }^{3}$ Whether or not tropes are objects inhering in themselves is a further question, which can be decided only on the basis of an analysis of inherence (the object's having a property) the specific trope theory adopts. The different trope theories analyse inherence (the relation between a property trope and the object possessing the trope) in different ways, cf. Keinänen $(2011$, sec. 3,2018$)$ for an overview.
} 
Mumford 2006, 475ff.). We need to assume that at least these properties to provide a sufficient account of the nature of concrete entities.

Therefore, we consider it the least controversial commitment to begin with, both in epistemic and ontological terms, that there are tropes falling under the determinables of the basic physical quantities. Here, we follow the lead of Campbell's (1990, 24-5) sparse theory of tropes and assume that there are tropes of a few basic physical quantitative kinds. ${ }^{4}$ In continuation, we concentrate on giving an account of the natural kinds of basic physical quantity tropes. It is coherent with our sparse conception of tropes that there are undiscovered quantity tropes of new kinds possessing some unexpected features.

We are not committed to the existence of quality tropes, or tropes analogous to qualities. Paradigmatic examples of qualities, determinate colours and shapes are not good candidates for tropes. Firstly, the contemporary metaphysicians of colour have provided good reasons to reject colours as (simple or complex) properties of objects. ${ }^{5}$ Secondly, shapes are (at best) regarded as properties of complex objects reducible to objects having proper parts in certain spatial relations. There is no reason to introduce shape tropes in addition to this explanation: the (sufficiently) fundamental proper parts of physical objects are not shaped, rigid bodies; rather, they are "powerful particulars" with causal powers sufficient to explain the shape properties of more complex objects (Keinänen 2011, sec. 4). In addition to these a posteriori-oriented considerations, we present more general metaphysical reasons for the rejection of quality tropes in trope theory in section 2.6.

\footnotetext{
${ }^{4}$ The precursors of Campbell's sparse theory of tropes are Armstrong's (1978) a posteriori realism about universals and Lewis's (1983) conception of sparse properties.

${ }^{5} \mathrm{Cf}$. Maund 2012, secs. 6.2-3. One difficult problem that any such identification of colours with properties would face is the problem of metamerism: we can observe a single colour on the basis of an object reflecting light having wavelengths of indefinitely many different alternative combinations.
} 


\subsection{The standard view of tropes of the same kind}

According to what could be called the standard view of tropes being of the same kind, determinate kinds of tropes are accounted for in terms of exact resemblance. In this account, exact resemblance between distinct tropes $t_{1}$ and $t_{2}$ is considered a basic internal relation. The fundamental idea behind the conception of internal relations which we adopt here is that entities can be related without there being any entity (i.e. relation) relating them. Since internal relations are not entities, they do not constitute "an addition of being" (Simons 2003; Lowe 2006, 46; cf. Betti 2015, 89).

For our present purposes, it suffices to stipulate that basic internal relation $\mathrm{R}$ holds between its relata $a$ and $b$ if and only if (i) necessarily, Rab if and only if $a$ and $b$ exist; (ii) R holds merely because of the existence of its relata. According to clause (i), necessarily, if the relata of basic internal relation $\mathrm{R}$ exist, $\mathrm{R}$ holds between the relata. ${ }^{6}$ Moreover, we assume that no relation can hold without the existence of its relata. Therefore, we accept (i) in a bi-conditional form. Here, we confine ourselves to state that a basic internal relation holds solely because of the existence of its relata, and we have clarified this condition elsewhere (cf. Keinänen et al. 2017, sec. 2).

In order to illustrate the conception of exact resemblance as a basic internal relation, let us now consider two $1 \mathrm{~kg}$ tropes $t_{1}$ and $t_{2}$. According to the standard view, these tropes are exactly resembling. The exact resemblance between $t_{1}$ and $t_{2}$ fulfils both conditions (i) and (ii) for a basic internal relation. First, necessarily, if $t_{1}$ and $t_{2}$ exist, these tropes are exactly resembling. Secondly, the converse holds; necessarily, if $t_{1}$ and $t_{2}$ are connected by exact resemblance, tropes $t_{1}$ and $t_{2}$ exist. Finally, tropes $t_{1}$ and $t_{2}$ are exactly resembling solely because of their existence as thin natures. If we want to express this in terms of the theory of

\footnotetext{
${ }^{6}$ Cf. Mulligan (1998, 344-5) and Simons (2003) for a similar conception of internal relations. The core idea of this conception is that the existence of the relata is sufficient for an internal relation to obtain.
} 
truthmaking, tropes $t_{1}$ and $t_{2}$ are jointly the minimal truthmaker of the proposition that $t_{1}$ and $t_{2}$ resemble exactly (cf. O'Conaill and Tahko 2016).

Prima facie, we have here a straightforward account of tropes belonging to the same kind. First, tropes are of the same determinate kind if and only if they are all mutually connected by the basic internal relation of exact resemblance. Consider, for instance, $1 \mathrm{~kg}$ tropes. Any $1 \mathrm{~kg}$ trope $u$ can serve as a paradigm. Any trope $t$ is then a $1 \mathrm{~kg}$ trope if and only if $t$ exactly resembles $u$.

All mutually exactly resembling or less than exactly resembling tropes appear to fall under the same highest determinable (e.g. mass, electric charge, length). Trope theorists usually claim that the tropes falling under a determinable resemble "in different degrees" (cf. Campbell 1990, 30ff.; Maurin 2002, 80ff.). Nevertheless, one can attempt to clarify the talk about "resemblance in different degrees" in the special case of quantity tropes. All quantity tropes falling under the same determinable are either exactly resembling or mutually connected by some necessary relation of quantitative distance. The relations of quantitative distance are expressed by means of the unit of the quantity under consideration. For instance, any two distinct mass tropes $t_{1}$ and $t_{2}$ are either exactly resembling or $t_{1}$ is $\mathrm{n}$ units (i.e. $\mathrm{n}$ times some unit of mass) greater than $t_{2}$, or vice versa. In order to incorporate this account of "inexact resemblances" into the nominalist account of kinds of tropes, the trope theorist should consider these quantitative distances basic internal relations. They should be considered to hold because of the tropes being the particular natures they are.

In the next section, we formulate a new trope nominalist account of basic internal relations between tropes that provides an explanation of their falling under determinate and determinable quantities. ${ }^{7}$ The basic internal relations introduced in our conception uniquely

\footnotetext{
${ }^{7}$ The notion of determinable is sometimes used to refer to all "general kinds", which include ontological categories and the kinds based on some shared formal ontological feature like the kind of dependent existents. The membership of an entity in these general kinds is, at least in a large part, determined by the formal
} 
characterize quantity tropes (as contrasted with the possible quality tropes). Second, our conception eliminates the irreducible reference to determinables. Quantitative distances between tropes are also accounted for by means of these basic internal relations. ${ }^{8}$

\subsection{The relations of proportion and order between tropes}

We dispense with all primitive resemblances between quantity tropes, exact or inexact. Our main thesis is that, as determinate particular natures, quantity tropes falling under a single determinable (e.g. rest mass) are all mutually connected by two different kinds of necessary relation. The first are the different relations of proportion, which can be expressed by means of real numbers. ${ }^{9}$ The second kind of relation is the relation of order, which generates a non-strict total order among the tropes falling under a determinable (e.g. rest mass). In order to properly capture the force of our approach, it is important to emphasize two things. Firstly, due to being determined by tropes (as thin natures), the relations of proportion and order remain invariant in all changes of the unit of any quantity. By contrast, the absolute quantitative values associated with tropes (like $1 \mathrm{~kg}$ or e charge) are dependent on the conventional choice of the unit. Secondly, although we speak about certain kinds of tropes (e.g. rest mass tropes or $1 \mathrm{~kg}$ tropes), the relations of proportion and order between tropes uniquely determine the determinates and determinables under which the different quantity tropes fall.

In order to illustrate our approach, let us consider the tropes falling under a single determinable, such as rest mass. All rest mass tropes are mutually connected by the necessary

ontological relations it bears to other entities. We will not discuss these general kinds here. See Hakkarainen \& Keinänen (2017) for the distinction between formal ontological relations and the internal relations (like proportions) based on the natures of entities.

${ }^{8}$ Keith Campbell (1990, secs. 4.3-4) has also presented a trope nominalist account of internal relations between inexactly similar quantity tropes falling under a determinable, cf. Keinänen et al. (2017, sec. 4).

${ }^{9}$ The present suggestion to employ the relations of proportion to spell out the relations between determinate quantities is inspired by Bigelow and Pargetter's (1990, 55-62) theory. Bigelow and Pargetter introduce proportions as second-degree relation universals - i.e. relations between relation universals. By contrast, proportions are considered here as basic internal relations between quantity tropes. 
relations of proportion and order. In order to facilitate the presentation, let us assume that there are both $1 \mathrm{~kg}$ tropes and $2 \mathrm{~kg}$ tropes. (Better a posteriori examples would be the rest mass tropes possessed by elementary micro-particles, such as the rest mass trope of an electron.)

Let $t_{3}$ be a $2 \mathrm{~kg}$ trope and $t_{4}$ be a $1 \mathrm{~kg}$ trope. Necessarily, if tropes $t_{3}$ and $t_{4}$ exist, proposition [1] is true:

[1]: Trope $t_{3}$ is in $2: 1$ proportion to trope $t_{4}$

Second, all rest mass tropes are connected by equal to or greater than, which we call the relation of order. Necessarily, if tropes $t_{3}$ and $t_{4}$ exist, proposition [2] is true:

[2]: Trope $t_{3}$ is equal to or greater than trope $t_{4}$.

One can state the connection between these two relations as follows: if the relation of order holds only in one direction (like in the case of tropes $t_{4}$ and $t_{3}$ ), we must express the proportions between rest mass tropes in a certain order that corresponds to how tropes are arranged by the relation of order (like in sentence [1]). By contrast, if the relation of order holds in both directions (tropes are equal in order), the tropes are connected by the relation of 1:1 proportion. To illustrate, let us consider two $1 \mathrm{~kg}$ tropes $t_{4}$ and $t_{5}$. Necessarily, if tropes $t_{4}$ and $t_{5}$ exist, proposition [3] is true:

[3]: Trope $t_{4}$ is in $1: 1$ proportion to trope $t_{5}$.

Tropes fall under the same determinate if and only if they are connected by the relation of $1: 1$ proportion. By the same token, these tropes are equal in order. Thus, our approach replaces talk 
about "exactly resembling" quantity tropes with talk about tropes connected by the relation of 1:1 proportion. Hence, the notion of exact resemblance does not play any role in our theory (even considered as a derived notion).

It is now possible to choose the unit for rest mass: we can take any of the rest masses of the rest mass tropes mutually connected by 1:1 proportion and select it as the unit. After the unit has been chosen, we can specify the quantitative distances ("inexact resemblances") between the distinct rest mass tropes by means of the relations of proportion and order. In our account, quantitative distances are derivative relations between tropes and, unlike the relations of proportion, they are dependent on the conventional choice of the unit. Since we can specify quantitative distances with the help of tropes selected as a unit, we need no reference to the determinable quantity (rest mass).

Due to being objective and determined by tropes (as thin natures), the relations of proportion and order are invariant - they remain invariant in all changes of the unit of any quantity. Consider again rest mass tropes. For instance, whatever quantitative value U our unit of rest mass gives to trope $t_{3}$, trope $t_{4}$ is a $1 / 2 \mathrm{U}$ trope, since these tropes stand in the relation of 2:1 proportion to each other. The relations of proportion between rest mass tropes are invariant. Similarly, trope $t_{3}$ is equal to or greater than $t_{4}$ (but not vice versa) because the relation of order remains invariant.

The relations of proportion are to be contrasted with the proportion-like relations the rest mass tropes appear to bear to the tropes falling under the other highest determinables (e.g. charge tropes) or to some other entities (e.g. numbers). These relations are not invariant because they are not fixed by tropes but by our choice of units. They would change depending on our decision: for instance, if one changes the unit of rest mass or electric charge in a suitable way, the proportion-like relations between rest mass tropes and electric charge tropes would 
also change. Similarly, the proportion-like relations between rest mass tropes and numbers would change depending on the choice of the unit for rest mass.

Hence, all rest mass tropes are mutually connected by the relations of proportion and the relation of order, which are directly determined by tropes. They do not bear these necessary relations to any other entity. In the determination of tropes falling under the determinable rest mass, we do not need to assume kinds of tropes. Since tropes are thin particular natures, any rest mass trope (particular rest mass) $u$ may serve as a paradigm. Trope $t$ is a rest mass trope if and only if $t$ is in some relation of proportion and in the relation order to trope $u$. Similarly, the tropes falling under a single determinate mass (e.g. $1 \mathrm{~kg}$ tropes) are determined by dint of the relation of $1: 1$ proportion. Let $v$ be any $1 \mathrm{~kg}$ trope serving as a paradigm. Trope $t$ is a $1 \mathrm{~kg}$ trope if and only if $t$ is in relation of $1: 1$ proportion to $v$. These tropes are also equal to each other.

With slight modifications, the proposed account can provide an account of the "resemblances" among the tropes of different basic physical quantities. It seems that all basic monadic quantities other than rest mass come in natural units (or are quantized) and have both positive and negative values. The best-known example is electric charge, which has both negative (e.g. -e, -e/3, -2e/3) and positive (e.g. e, e/3, 2/3e) basic units. ${ }^{10}$ The trope theorists adopting an empirical conception of properties postulate tropes to determine at least the electric charges of the fundamental particles. ${ }^{11}$

In order to extend our theory to electric charge tropes, one must assume that the relations of proportion can have both positive and negative values. Although the positive and negative proportions are fixed mind-independently by tropes, one has complete freedom to stipulate which of the values of charge are negative and which positive while introducing the

\footnotetext{
${ }^{10}$ These basic units include both the charges of leptons and their anti-particles as well as the charges of quarks and anti-quarks.

${ }^{11}$ According to some trope theorists (e.g., Simons 1994, 1998), only simple objects are trope bundles. By contrast, Keith Campbell (1990) postulates complex tropes, which are properties of complex objects.
} 
unit of electric charge. One possible choice for the unit is the proton charge e. Moreover, all charge tropes are mutually connected by the relation of order, which is a total order. However, it is up to the conventionally chosen unit of electric charge whether this is interpreted as a "lesser than or equal to" or "greater than or equal to" relation. Like in the case of mass tropes, an arbitrary charge trope $u$ can serve as a paradigm. There is no need for an irreducible reference to the determinable. Trope $t$ is a charge trope if and only if $t$ bears some relation of (positive or negative) proportion and the relation of order to $u$.

According to our theory, the (determinate or determinable) quantity D under which a quantity trope falls is determined by tropes as particular natures. There is no need to assume kinds of tropes as particular or universal entities. Any trope $u$ can serve as a paradigm among the $\mathrm{D}$ tropes. Let $\mathrm{D}$ be a determinable kind of quantity tropes (a similar account may be provided of the tropes belonging to a determinate kind). Trope $t$ falls under the highest quantitative determinable $\mathrm{D}$ if and only if $t$ is in some (positive or negative) proportion and in the relation of order to $u$. By contrast, the quantity tropes falling under distinct highest determinables are not connected by the relations of proportion or by the relation of order. The relation of order together with the different relations of proportion divide quantity tropes into distinct and mutually exclusive groups (equivalence classes). Therefore, every trope falls under exactly one highest determinable.

Although our theory replaces the standard view of tropes belonging to the same determinate/determinable kind, it relies on the standard conception of tropes as thin particular natures. These natures are quantitative and determine the relations of proportion and order between tropes. Paradigm tropes are only required to fix our reference to the mutually internally related tropes, which belong to the same determinate/determinable kind. 
Prima facie, our account is applicable to such monadic physical quantities as rest masses, electric charges, and spin quantum numbers. ${ }^{12}$ However, its general applicability is an open question. For instance, if there are such entities as monadic tropes of colour charge, the necessary relations between them would need further work. ${ }^{13}$ Similarly, we must leave the generalization of the present approach to relational or relation-like tropes (e.g. tropes of spacetime interval, if there are such entities) for another occasion. There is, however, one special case of monadic tropes that must be taken into account in the present paper.

\subsection{The special case of zero-value physical quantities}

According to some metaphysicians, there exist zero-value physical quantities that are not to be confused with the mere absence of these quantities. Balashov (1999) argues that the best metaphysical account of certain fundamental physical properties needs to incorporate zero-value quantitative properties such as zero electric charge and zero spin. There would thus be a real difference between the case where particle $a$ has zero electric charge and the case where $a$ has no electric charge. Assuming this is correct, it might seem that our theory as presented thus far is unable to account for the falling under the same determinate quantity of, say, two zero charge tropes $t$ and $u$. This appearance is due to the mathematical fact that the proportion 0:0 is not defined.

Should zero-value physical quantities exist, they are accommodated by our theory as follows. Zero-charge tropes $t$ and $u$ are connected by the relation of order in the obvious way: $t$ is equal to or greater than $u$, and $u$ is equal to or greater than $t$. Thus, $t$ and $u$, like all other zero-charge tropes, form a natural equivalence class due to being ordered this way. Hence, in the case of zero-quantity tropes, proportions cannot be relied upon in explaining their

\footnotetext{
${ }^{12}$ Cf. Balashov (1999) and Morganti (2009) for useful discussions of basic monadic physical quantities.

${ }^{13}$ Cf. Balashov $(1999,274 f f$.$) and Maudlin (2007,86 \mathrm{ff}$.) for discussions of colour charge.
} 
falling under the same determinate quantity; rather, the relation of order is called upon. The same account can obviously be given for non-zero-quantity tropes as well instead of invoking $1: 1$ proportions.

Moreover, zero-charge tropes $t$ and $u$ are connected by the relation of order and the asymmetric relation of zero-proportion to any non-zero-charge trope $v$. As with non-zero-charge tropes, the relations of proportion are invoked in the account of quantitative distances between a zero-charge trope and other charge tropes. Here, it must be noted that the distances between zero-charge tropes and non-zero-charge tropes do not fall out of the theory quite in the simple way as those between non-zero-charge tropes, since the proportions of charge between, say, zero and $-e$ would be the same as that between zero and $-e / 3$. What differentiates these quantitative distances, as in all such cases of non-zero quantities, is the proportions between the non-zero quantities of the determinable in question, in our example

the proportions between the non-zero-charge tropes. As there is "a quantitative distance" of $-2 e / 3$ between an $-e$ trope and $-\mathrm{e} / 3$ trope, their respective distances to a zero charge trope are different.

\subsection{Proportion and order as basic internal relations}

It is fairly straightforward to argue that the relations of proportion and order between tropes are basic internal relations. In other words, tropes are in these relations as the thin natures they are. Consider, for instance, the relation of the 3:1 proportion between -e charge trope $t_{6}$ and -e/3 charge trope $t_{7}$. Proposition [4] is true:

[4]: Trope $t_{6}$ is in 3:1 proportion to trope $t_{7}$. 
First, necessarily, $t_{6}$ is in the relation of $3: 1$ proportion to $t_{7}$ if and only if $t_{6}$ and $t_{7}$ exist. Second, the proportion relation holds solely because of the existence of $t_{6}$ and $t_{7}$ : given that $t_{6}$ and $t_{7}$ exist as the tropes they are, that is, a particular -e charge and a particular -e/3 charge, they are in this proportion relation. To put this in terms of truthmaking, tropes $t_{6}$ and $t_{7}$ are jointly the minimal truthmaker of proposition [4]. These claims are easily generalized to all proportion relations between quantity tropes (e.g. to those described by [1] and [3]).

We can argue in a similar way that the relation of order is a basic internal relation between quantity tropes. Again, tropes $t_{3}$ and $t_{4}$ are jointly the minimal truthmaker of proposition [2]. ${ }^{14}$ The only further complication results from the fact that, if considered as a specific "equal to or greater than" or "equal to or smaller than" relation, the direction of the relation of order is, at least in many cases, dependent on our conventions.

\subsection{Our Theory and the Standard View}

In this section, we shall show the superiority of our account to the standard view by a disjunctive, non-demonstrative argument. In this argument, we make the background assumption that there is an intuitive, ready-to-hand distinction between qualities and quantities. As we noted in section 2.1, the best a posteriori examples of tropes fall under the basic physical quantitative determinables. Therefore, we may set aside the alternative that there are only quality tropes and the disjunctive argument consists of only two horns: either there are only quantity tropes or there are both quantity and quality tropes. The argument concludes that our theory has better explanatory power regarding the kinds of tropes than the standard view. Hence, our theory is superior and should be endorsed rather than the standard view.

\footnotetext{
${ }^{14}$ We remain uncommitted to non-actual possible worlds or merely possible entities. However, if one wishes to assume merely possible quantity tropes of actual kind $\mathrm{K}$, then they are as particular natures in certain basic internal relations of proportion and order to the actual tropes of this kind. Similarly, relative to the fiction, fictional tropes of kind $\mathrm{K}$ would bear these relations to the actual tropes of this kind.
} 
In the first quantity horn, let us suppose for the sake of argument that there are only quantity tropes. As we noted in section 2.3, the basic internal relations of proportion and order uniquely specify the determinate or determinable quantity under which a given trope falls. These basic internal relations are determined by tropes, and in them, there is no recourse to the determinables or determinates.

By contrast, as we saw in section 2.2, the standard view does not have similar means of specifying the tropes falling under a quantitative determinable. Since she uses inexact resemblance here, the advocate of the standard view sticks to the "degrees of resemblance". The best thing she can do is to present the degrees of resemblance between quantity tropes in terms of quantitative distance: the tropes falling under a determinable (say, rest mass) are either exactly similar or related by some relation of quantitative distance. One must give the quantitative distance between two rest mass tropes $v$ and $w$ by recourse to the unit of mass: 3 $\mathrm{kg}$ trope $v$ is $1 \mathrm{~kg}$ greater than $2 \mathrm{~kg}$ trope $w$. Unlike our approach presented in section 2.3 , the standard approach should take this quantitative distance ("inexact resemblance") as an unanalysable basic internal relation between tropes $v$ and $w$.

The main problem with this view is that the reference to the unit contains an implicit reference to the determinable (mass): trope $v$ is by a certain unit of mass $U$ greater than trope $w$. More generally, if taken as primitive, a precise characterization of the relations of quantitative distance requires a recourse to the determinable with respect to which the quantity tropes are inexactly resembling. By the same token, the explanation of why tropes fall under the same determinable (rest mass) would require a recourse to the very determinable (rest mass). Therefore, the attempt to construe determinables by means of tropes connected by the relations of quantitative distance would be circular.

In the conception relying on primitive quantitative distances, the only clear way to avoid circularity in the determination of tropes falling under a determinable is to take the 
determinable property as an additional entity, which the tropes instantiate. For instance, tropes are in some quantitative distance with respect to mass because they instantiate the determinable property mass. Because of being instantiated by distinct tropes, the determinable would be naturally considered as a property universal (cf. Armstrong 1978, 45). ${ }^{15}$ Since this universal/determinable property would be an additional entity to the tropes, the relation of inexact resemblance holding between these tropes would not be a basic internal relation. Its holding requires the existence of an additional entity to the tropes standing in the relation.

Hence, it is a general problem for the standard view that inexact resemblances considered as quantitative distances are not good candidates for basic internal relations. Our theory is superior to the standard view because the relations of proportion and order can be specified without recourse to the determinable. Even if the standard view was able to account for the determinate kinds of quantity tropes (as we are), it still would not provide a satisfactory account of the determinable kinds. This is the conclusion of the first horn of the disjunctive argument.

The second horn of the argument is more complicated, as we assume, for the sake of the argument again, that there are both quality and quantity tropes. We already presented empirically motivated considerations against taking particular shapes and colours as serious candidates for tropes. In this section, we argue that these quality tropes are problematic for a more general reason: the determinate colour or shape tropes occupy certain regions of space having a certain shape and size. The occupation of different kinds of regions must be based on an intrinsic difference among otherwise "exactly similar" quality tropes. Therefore, in many cases, there is some intrinsic difference between the quality tropes falling under exactly the

\footnotetext{
${ }^{15}$ For instance, Ingvar Johansson (2000) construes determinable properties as determinable universals, which provide the quantitative distances between determinate properties. In a trope version of this view, tropes instantiate determinable universals, which determine the quantitative distances between the tropes falling under a determinable.
} 
same determinate. This frustrates the standard conception of quality tropes falling under a determinate as thin natures that occur in non-relativized relations of exact resemblance.

Before proceeding, we provide a brief explanation of why the same problem does not concern quantity tropes. As we already noted in section 2.1, complex physical objects are constituted by "powerful particulars". Fundamental monadic quantities are properties of a powerful particular (like electric charges) and contribute to determining its causal powers to act in a certain way (Keinänen 2011, 445 ff.). Powerful particulars do not have determinate shapes or sizes as their additional monadic properties. Rather, they have a (not necessarily point-like) centre of influence as their location. Powerful particulars might be considered to have approximate sizes determined by their causal powers and the causal powers of other powerful particulars. Quantity tropes, which constitute a powerful particular, are concrete and have the centre of influence of the powerful particular as their location. However, they do not have different sizes (or shapes). They do not have a set of causal powers, on the basis of which we could assign them a size (or a shape) as a derivative feature. ${ }^{16}$ Rather, they (partially) determine the causal powers of a powerful particular.

By contrast, if there are quality tropes, they occupy some extended regions of space. Consider, for instance, shape tropes such as disc-shaped trope $d$. Necessarily, if disc-shaped trope $d$ exists, it is a disc-shaped trope of some determinate size. Thus, if there are shape tropes, there must also be size tropes since each determinate shape trope is some determinate size trope (the "is of identity"; Giberman 2014, 457-8). Similarly, if there are colour tropes, it is highly plausible that there are extended colour tropes, since colours spread

\footnotetext{
${ }^{16}$ Cf. Harré (1970, ch.12) and Keinänen (2011, sec. 4) for more on powerful particulars. Note that in the case of a powerful particular, having size and shape is not a direct consequence of having a (determinate/approximate) location. For an object to have size and shape would require it to have some borders. However, an object can have borders only on the basis of how it interacts with other objects; merely having a location is not sufficient. These ways of interaction are determined by its causal powers and the causal powers of other objects. On formal features of tropes, such as their numerical identity and particularity, cf. Hakkarainen and Keinänen (2017, sec. 651ff.).
} 
out over surfaces. Given the existence of extended colour tropes, they are determinate formed volumes: for instance, the crimson ball-shaped trope of a ball.

Thus, it seems that determinate shape and colour tropes are complex natures that have separate "aspects" in a numerically identical entity - at least a determinate shape and size, or a determinate colour, shape, and size. Assuming that there are colour tropes, there are colour tropes that are also determinate shape tropes. Given that the ball is wholly crimson and its entire crimsonness is a trope, the crimson trope is ball-shaped, that is, also a ball-shape trope. According to Campbell's (1981) earlier conception of tropes, both shape and size are essential to every trope but are not themselves separate tropes. The basic idea here is that every trope constitutes a formed volume - e.g. the crimsonness of a specific region of space. An object is a sum of co-located tropes in that same region. The purpose of Campbell's move is to explain how a quality trope - like the crimsonness trope of a ball - can exist as co-located with a certain shaped object but avoid the introduction of distinct shape tropes. In Campbell's trope theory, co-location is a necessary condition for tropes being properties of an object. Colour tropes must already be determinate shapes and sizes in order to be co-located with certain other tropes, which are parts of a single object. Therefore, there seems to be no need to introduce shape or size tropes as additional entities.

The combinations of determinate shapes and sizes, and determinate colours, shapes, and sizes vary. What can only be required is that a colour trope is some shape and size, and that a shape is some size, etc. Given the notions of the standard view, a crimson disc-shaped trope and a crimson ball-shaped trope resemble exactly (with respect to colour). By the same token, they do not resemble exactly (with respect to shape). Similarly, if there are shape tropes, a disc-shaped small trope and a disc-shaped big trope both exactly resemble with respect to shape but do not exactly resemble with respect to size. The crucial point here is that it is one and the same trope $t$ that is both crimson and disc-shaped and another trope $u$ that is crimson 
and ball-shaped. If trope $t$ is numerically identical with a particular crimsonness and $u$ is another particular crimsonness, $t$ and $u$ should be considered to resemble exactly. However, due to being different shapes, they do not resemble exactly, which contradicts the first claim.

One can avoid this contradiction by relativizing the relation of exact resemblance of the standard view to determinables. The crimson disc-shaped trope and the crimson ball-shaped trope resemble exactly in respect of colour but not in respect of shape. Nevertheless, if not taken as further analysable, this kind of relativized relation of exact resemblance seems to hold because the tropes related by the relation instantiate the respective determinables (colour, shape). Hence, the standard view, when it is applied to the determinate kinds of quality tropes, either faces a contradiction or assumes determinables rather than explains tropes falling under a determinate by means of basic internal relations between tropes.

In his Abstract Particulars (1990), Campbell rejects his earlier view of tropes as formed volumes. He introduces "quasi-tropes", which spatial tropes such as shapes and sizes are, in order to maintain that tropes are thin natures $(1990,72-3,91)$. Quasi-tropes are numerically distinct from "genuine tropes" (ibid. 68, 91): "The shape of a coloured, or solid, or salty, or rusty, formed volume is an additional trope in its own right" (ibid. 66). Genuine tropes are numerically distinct from space, whose non-numerically distinct sub-regions or pseudo-parts - "place tropes" - are quasi-tropes (ibid. 66, 148, 152). Space itself is "a real, non-relational entity" (ibid. 66). The relation of compresence holds between mutually co-located tropes and a place trope (ibid 68-9, 94).

Since quality tropes such as colours are numerically distinct from spatial quasi-tropes, it might appear that they can resemble exactly without the need for relativizing the exact resemblance to determinables. Nonetheless, this appearance is only a mirage. According to Campbell, the "boundaries [of a spatial quasi-trope] are fixed by the presence of colour, solidity or whatever" (ibid 68-9). Even more tellingly, he states: 
As for the shapes, so far from them being merely ideal, the world is full of innumerable specimens of every possible variety. They overlap and intermingle because space has no intrinsic boundaries. Because boundaries in space need to be drawn rather than revealed, it is perhaps best to view individual specimens of each of the shapes as quasi-tropes rather than as genuine tropes. (Ibid. 91)

These two quotations strongly suggest that Campbell's mature view is that genuine tropes delineate quasi-tropes in space. Indeed, he thinks that each colour trope must have at least a spatial location, and most of them a shape (ibid. 70).

Since the relation of compresence holds between colour tropes or other ordinary quality tropes and a spatial quasi-trope (place trope), quality tropes are co-located with a quasi-trope. Thus, what is involved in a quality trope is both a generic disposition to delineate a quasi-trope and co-location with some quasi-trope (at every moment of the trope's existence). Crimson trope $t$, for instance, delineates, say, a disc-shaped place of some size at every moment of its existence, whereas crimson trope $u$ delineates a ball-shaped place. Moreover, both $t$ and $u$ are co-located with the corresponding place tropes. The general picture is slightly more complicated than in Campbell's earlier account: a colour trope can be co-located with different kinds of place tropes at different moments. However, quality tropes have non-numerically distinct aspects at every moment of their existence - such as a determinate colour and a determinate shape. Again, two distinct tropes can, for example, exactly resemble with respect to colour and differ with respect to shape, like the aforementioned tropes $t$ and $u$. 
Hence, also in Campbell's later account, the determinable kinds of tropes are presupposed rather than explained away on the pain of contradiction. ${ }^{17}$ Of the recent trope theorists, both Ehring (2011) and Giberman (2014) offer an extensive discussion of quality tropes. Because Ehring is a natural kind trope nominalist, the present problem does not directly concern Ehring's views. Giberman $(2014,458 \mathrm{ff}$.) adopts a position similar to Campbell's earlier view and identifies ordinary tropes with their shapes, sizes, and durations. According to Giberman, these shapes, sizes, and durations are tropes identical to the ordinary, "characterizing tropes" (determinate colours or masses, etc.), but not identical to their locations. Despite providing a new trope bundle theory of objects, Giberman does not give any explanation of how tropes can be exactly similar in respect of (say) colour but fail to be similar in respect of shape without relativizing these similarities to determinables. Thus, the problem remains.

In section 2.1, we provided empirically motivated considerations to doubt the existence of the paradigmatic kinds of quality tropes, namely shapes and colours. In this section, we assumed, for the sake of argument, that there are quality tropes. We argued that since quality tropes usually have different spatial aspects (such as shapes and sizes), there are intrinsic differences among the otherwise exactly similar quality tropes. Therefore, quality tropes may stand in different relations of exact similarity, which must be relativized to different determinables (e.g. colour, shape, size) in order to avoid a contradiction. This frustrates the standard trope nominalist attempt to offer an account of quality tropes belonging to a determinate kind. As argued in this section, no recent representative of the standard trope nominalism has been able to resolve this difficulty.

\footnotetext{
${ }^{17}$ Notice that one cannot defend Campbell against this objection by pointing out that ultimately he does not believe in colour tropes (1990, ch. 6.11). The objection occurs in the second horn of our argument when we suppose the existence of quality tropes, the paradigmatic example of which are colour tropes.
} 
Thus, the standard view does not provide any adequate trope nominalist account of quality tropes belonging to the determinate kinds. Rather, the standard view presupposes the determinable kinds of quality tropes in its account. Hence, it does not have any advantage over our theory in the second horn. As we are able to account for the quantity tropes belonging to determinable kinds, our theory has better explanatory power both in this horn and the first quantity horn that are exhaustive possibilities in our argument. The conclusion of our disjunctive argument is therefore that our theory is superior in explanatory power with regard to tropes belonging to the same kind than the standard view. Consequently, our theory should be endorsed rather than the standard view.

\section{Kinds of Tropes}

While in the previous section we discussed the relations determining that tropes are of the same determinate or determinable kind, in the present section, our focus will be on the ontological categorization of these kinds. If they are entities in their own right and numerically distinct from tropes, what is their ontological category? If they are not distinct entities, should we then say that they are tropes or eliminate them altogether from our ontology? First, we shall argue that none of the reductive accounts presented in the current trope literature or the Fregean abstraction view is acceptable to a trope theorist who wants to restrict her ontological commitments to tropes. After that, we will propose our eliminative account that kinds of tropes are not identified with anything: strictly speaking, there are no determinate kinds of tropes or shared determinate properties, whether considered as universals or particulars. Similarly, there are no determinable kinds of tropes. The trope theorist need not identify determinate or determinable properties (or determinate or determinable kinds of tropes) with anything.

Before we continue, let us make a clarification. We shall talk about determinate or determinable "kinds of tropes" and "properties" interchangeably. The distinction between 
kinds of tropes and property universals is ontologically significant for the realists concerning universals and for discussing realist views. By contrast, trope theorists, even if aspiring to a reductive analysis of determinate properties, have a strong motivation to treat kinds of tropes and shared properties in a similar manner.

\subsection{Proposed Accounts}

According to some influential state-of-the-art presentations, trope theorists identify determinate properties ("property universals") with classes or sets of exactly resembling tropes (Lewis 1986, sec. 1.5; Armstrong 1989, 120-1; Oliver 1996). Whether or not this has been a majority view among trope theorists, it has not been unanimously accepted. There are at least four alternative reductive accounts of kinds of tropes that trope nominalists adopt or at least consider. The fifth proposed alternative may be called the abstraction account of the kinds of tropes. Since the advocates of this view may have different kinds of commitments to universals or particulars constructed from tropes, we do not classify it as a reductive account.

(1) A.R.J. Fisher (2018, sec. 3) defends a view of determinate universals or properties that reductively identifies determinate kinds of tropes with tropes, the reductive identificationist view. Fisher exploits a theory of theoretical terms developed by Lewis (1970) and takes universals to be entities that play a certain role, say, the "R-role". The meaning of the term "universal" is fixed by the R-role. Similarly, another role, call it the "S-role", fixes the meaning of the term "particular". Moreover, the particular-universal distinction - that is, the distinction between the players of the S-role and the R-role - entails that universals satisfy the principle of the identity of indiscernibles, whereas particulars do not satisfy that principle (op. cit. 161). As a particular nature, every trope $t$ manifests (i.e. is an instance of) some occupant of the R-role. For example, -e charge trope $t$ manifests the determinate universal of -e charge. 
According to Fisher $(2018,160-1)$, tropes can now be taken as occupants of both the S- and R-roles by recourse to "the different rules of counting". As particular natures tropes are occupants of the S-role, any trope $u$ can also be taken as an occupant of the R-role by considering all tropes perfectly similar to $u$ identical to it. Assuming that $u$ is an -e charge trope, it is also universal of -e charge (it plays the corresponding R-role) if the perfect similarity of $u$ with certain other tropes is considered to entail numerical identity. There is no categorial distinction between property universals and tropes, but only a difference in the roles the same tropes are playing. Therefore, determinate kinds of tropes "are tropes" (op. cit. 161). Fisher's emphasis here means that he is speaking about "the is of identification" (ibid.). Indeed, the determinate kind -e charge is identified with any of the occupants of the universal -e charge role, which exist mind-independently. Thus, Fisher's account is reductionist rather than eliminativist since determinate kinds of tropes exist mind-independently (Fisher 2018, sec. 3).

Fisher is explicitly developing D.C. Williams" “Moderate Realism” (1986, 8-10), which inspired Campbell's "Painless Realism" (1990, 43-5). As well as in Fisher's account, according to Moderate and Painless Realism, the basis for the determinate kinds of tropes consists of the basic internal relations of exact resemblance among tropes. Because of their close affinity to Fisher's position, there is no need to discuss Moderate and Painless Realism in detail.

(2) In his "On the Elements of Being I", Williams advocated the identification of "property universals" (determinate kinds of tropes) with the sets of exactly resembling tropes (Williams 1953a, 6). According to this view, each kind is an abstract individual categorially distinct from its members - that is, tropes. The set account, which has been made widely known by the above-mentioned general presentations, has been later endorsed by early Campbell (1981, sec. 6). 
(3) According to Giberman (2014, 454-5), properties such as charge are resemblance classes of tropes, which can be interpreted as sets or spatio-temporally located pluralities. Assuming the latter reading, Giberman claims that every determinate kind is a plurality of exactly resembling tropes. Since the existence of a plurality is nothing else but a collective existence of its members, a determinate kind is on this view identical to a plurality of tropes.

(4) In his “On the Elements of Being I”, Williams (1953a, 6) tentatively considers the identification of property universals with mereological sums of exactly resembling tropes. However, he rejects this alternative.

(5) In addition to these four reductive accounts, there are different abstraction views of the kinds of tropes. John Heil is a representative of such a view. He modifies Williams' Moderate Realism with Locke's doctrine of abstraction by partial consideration (Heil 2012, 102). However, since Heil's view is based on the conception of tropes as attributes of substances, we will not discuss it further here.

There is also a different Fregean sense in which properties can be considered as abstractions from exactly resembling tropes. In this conception, determinate properties are abstracta under the equivalence relation of exact resemblance between tropes. ${ }^{18}$ A determinate property (e.g. being $1 \mathrm{~kg}$ ) is a result of abstraction from the tropes mutually related by the equivalence relation of exact resemblance (1 kg tropes). Here, the key idea is to introduce an abstract "invariant" corresponding to any chosen plurality of exactly resembling tropes by means of the abstraction principle (cf. Simons 2008, 174; Rosen 2012, sec. 6). Our ability to consider a determinate property as a unity (a single universal or kind) is a result of our applying the abstraction principle to exactly resembling tropes. The results of this abstraction - that is,

\footnotetext{
${ }^{18}$ Simons $(2008,171-4)$ takes up the possibility of formulating a conceptualist or nominalist theory of universals by taking universals as Fregean abstracta. Here, this idea is applied to the kinds of tropes.
} 
invariants - are abstract in the standard metaphysical sense of being non-spatiotemporal (Simons 2008, 172, 174). Simons is explicitly neutral with regard to the mind-independent or mind-dependent existence of the results of this abstraction $(2008,172,174)$.

\subsection{Assessment of the proposals}

Although some metaphysicians of a nominalist persuasion (cf. Lewis 1986; Rodriguez-Pereyra 2002) postulate sets, we do not take it to be an attractive option for a trope nominalist to identify determinate/determinable properties with sets of tropes. First, sets are - or, at least standardly considered - abstract (non-spatiotemporal) individuals, whereas tropes are concrete. By introducing abstract individuals, trope theory would lose at least part of its initial appeal as a metaphysical system introducing only entities that have effects on the spatio-temporal world. ${ }^{19}$ Secondly, even if one would claim that sets are concrete (and perhaps located in the spatiotemporal positions of their members), they would still constitute a basic category of entities in addition to tropes. As a category, sets would be determined by the basic formal ontological relation of set-membership. Therefore, trope theory would be qualitatively less economical than initially considered (a one- or two-category ontology of tropes).

We leave the fourth reductive option (identification of properties with sums of tropes) without more detailed consideration partly because it has not been endorsed by any trope theorist, and partly because it is not prima facie plausible. For instance, a mereological sum of tropes is partly located in every region of space in which its parts are located. Therefore, unlike (universal) properties, it need not be "wholly present" in its every instance. Since pluralities also have a spatio-temporally scattered location in the locations of their members, exactly the same worry concerns the third option identifying a determinate kind with a plurality

\footnotetext{
${ }^{19} \mathrm{Cf}$. more general discussion of mathematical entities, e.g. Papineau 2016, sec. 1.8 .
} 
of exactly similar tropes. Furthermore, unlike mereological sums, pluralities are not individuals. Therefore, identifying determinate kinds with pluralities of tropes requires adopting a non-standard view of numerical identity as a one-to-many-relation. Adopting such a non-standard view is a theoretical cost. For these two reasons, it seems to be a less problematic alternative to try deal with the pluralities of resembling tropes without identifying them with kinds as individual entities.

Of the remaining options, Fisher's reductive identificationist view (option 1) and the Fregean abstraction view (option 4) are articulated in most detail. To start with the abstraction view, it can be applied to tropes standing in any equivalence relation to each other. We may assume the equivalence relation to be 1:1 proportion or being equal in order. Thus, for instance, one may claim that the following abstraction principle holds true:

[5]: Necessarily if tropes $t$ and $u$ stand in the relation of 1:1 proportion to each other, there is determinate kind $\mathrm{K}$ (e.g. -e charge) and $t$ and $u$ are instances of kind K (-e charge tropes).

As particular natures, tropes determine the kind $\mathrm{K}$, to which they belong. $\mathrm{K}$ would be an abstract individual (the property of -e charge), which is also in the relations of proportion and order to other abstract individuals (e.g. the property of e charge). The relations of proportion and order between kinds of tropes correspond to the respective relations between tropes (cf. Simons 2008, 173-4).

Nevertheless, the abstraction view goes beyond pure trope ontology by introducing abstract individuals, which cannot be reduced to (or constructed by) tropes. Therefore, it has exactly the same two problems as the view identifying properties with sets of 
exactly resembling tropes. An abstraction principle like [5] secures the existence of an abstract individual, provided that there are certain kinds of tropes in the equivalence relation of $1: 1$ proportion. However, abstraction principles introduce abstract individuals as additional entities, which also form an additional category (e.g. universal properties or concepts).

Since Fisher's reductive identificationist account attempts to identify kinds of tropes with tropes and does not introduce any other entities than tropes, it seems superior to the abstraction view. Fisher $(2018,159-60)$ points out that trope $t$ determines its determinate kind, $\mathrm{K}$. Moreover, trope $t$ is a (minimal) truthmaker of the corresponding statement that $t$ is of the $k i n d K$. Thus, the way in which Fisher deals with the "why question" (why does trope $t$ manifest the kind $\mathrm{K}$ ?) is in line with the widely accepted conception of tropes as particular natures. Nevertheless, the way in which he deals with the "how question" (how does trope $t$ manifest the kind $\mathrm{K}$ ?) turns out to be problematic.

For dialectical purposes, trope theorists are completely free to specify the "R-role", which lays down the role of universals in an ontological system containing tropes. This would include claims like "the identity of indiscernibles holds true of universals" and "universals are entities that are manifested (or instantiated) by tropes". Moreover, trope theorists can define terms like "qualitative identity" by means of the resources available in their category system.

Nevertheless, it is a highly problematic part of Fisher's account to take tropes as occupants of the R-role (cf. op. cit. 161). It might be part of a (dialectical) characterization of an ontology containing tropes that the identity of indiscernibles holds true of the potential occupants of the R-role. ${ }^{20}$ By contrast, the question of whether the R-role has any occupants is an issue independent of the specification of that role. The answer to this question depends on

\footnotetext{
${ }^{20}$ Daniel Giberman (2016) and Gonzalo Rodriguez-Pereyra (2017) have recently raised objections to distinguishing universals from particulars by means of the holding of the identity of indiscernibles.
} 
what different kinds of entities there are. If there are tropes, there are distinct entities related by 1:1 proportion (e.g. -e charge tropes) that are not identical. Thus, these -e charge tropes are not identical to a single -e charge trope $t$. We can, of course, conceive what it would mean if $t$ were identical to all these (alleged) tropes - they would be a single universal and a potential occupant of the R-role. But since all these -e charge tropes are distinct, it does not help to consider all the -e charge tropes identical to $t$. If there are no universals in addition to tropes, the R-role does not have any occupants. Therefore, one cannot develop a reductive theory of universals on the basis of taking tropes as the occupants of the R-role.

We can also look at the matter from a slightly different angle. Identity and distinctness are formal ontological relations, whereas in trope theory, 1:1 proportion (or exact similarity) concerns the nature of the related entities, namely tropes (cf. Hakkarainen and Keinänen 2017). One can make additional formal ontological assumptions about the identity of entities. For instance, one might claim that the tropes related by 1:1 proportion are identical. By making such an assumption, one can achieve one of two things. First, one might replace tropes with universals and reject the trope ontology. Second, one might go beyond pure trope theory by claiming that there is some different but ontologically acceptable sense in which the distinct tropes related by 1:1 proportion are identical. As a formal ontological relation, the "identity" is not just another way to express the 1:1 proportion between tropes.

\subsection{Our Proposal}

Trope theorists may have two general motives for involving kinds or ("universal") properties in their category system. One is the intuition that there is generality in the world, which requires explanation. Another motive would be to have a straightforward account of the reference of general terms. For instance, one might suggest that general terms like "-e charge" present in 
the statements of laws of nature refer to general entities. Nevertheless, considering the serious problems of the abstraction view and the different reductivist positions, we propose that the trope nominalist should adopt an eliminativist account by refusing to identify determinate/determinable kinds of tropes with anything. There are no determinate kinds of tropes (determinate properties) but only different pluralities of tropes standing in mutual basic internal relations of 1:1 proportion (or, in the case of zero quantities, in the basic internal relation of order in the way explained in section 2.4). Similarly, there are no determinable kinds of tropes but different pluralities of tropes standing in mutual basic internal relations of proportion and the relation of order. We need not ontologically categorize these kinds in any way because they do not exist.

Our eliminativist proposal is in line with the strict nominalist doctrine that there is no universality or generality in the world. Yet we are capable of general talk - talk about determinate properties and their mutual interrelations, for instance. The key for our eliminativist position is a meaning-theoretic account that denies the need to consider general terms as expressions referring to general entities (e.g. determinate properties). Thus, what is needed is some plausible account of the truth conditions of sentences that are used to make assertions about determinate/determinable properties and determinate or determinable kinds of tropes. To make such an account plausible, one would at least need a meaning-theoretic, or metasemantic, account of the determination of the semantic features of general terms like " 1 kg trope".

To develop such an account is beyond the scope of this paper. Therefore, we do not maintain that such an account would be in every respect superior to the semantic views that assume a direct reference to determinate/determinable properties. Our sole purpose is to illustrate how a metaphysically motivated eliminativist view can be combined with a feasible semantics of general terms. One possible strategy would be to give a theory of reference 
determination for kind predicates like "is a $1 \mathrm{~kg}$ trope". Let us say that a monadic predicate term "P" exactly applies (at time t) to a certain plurality of entities if and only if "P" applies to each of these entities and the entities in the plurality are the only entities to which "P" applies. ${ }^{21}$ Any $1 \mathrm{~kg}$ trope $u$ may serve as the paradigm or "sample" for the fixing of the application of the predicate "is a $1 \mathrm{~kg}$ trope". The sample trope $u$ would then contribute to the determination of the application conditions of the predicate in the obvious way. The kind term "is a $1 \mathrm{~kg}$ trope" exactly applies to the tropes that bear the relation of $1: 1$ proportion to trope $u$ (or, in the case of zero quantities, to tropes that are connected to $u$ by the relation of order as laid out in section 2.4). The reference of the determinable kind term "is a mass trope" can be similarly fixed by relying on the same sample trope $u$; in this case, "is a mass trope" would exactly apply to the tropes that bear some relation of proportion and the relation of order to trope $u$.

In order to illustrate how the attributions of determinate kinds to tropes are made true, let us consider proposition [6]:

[6]: $t_{4}$ is a $1 \mathrm{~kg}$ trope.

Tropes $t_{4}$ and $u$, which are connected by the relation of $1: 1$ proportion, jointly make true proposition [6]. However, assuming that $t_{4}$ and $u$ are distinct, they are not the minimal truthmaker. Rather, since $t_{4}$ could itself have been chosen as the paradigm trope, $t_{4}$ is the minimal truthmaker of [6]. In a similar way, we argue that $t_{4}$ is the minimal truthmaker of the proposition that $t_{4}$ is a mass trope.

\footnotetext{
${ }^{21}$ Notice that the reference of kind predicates does not have to be denotation (singular reference) but the divided reference of application.
} 
Unlike the current reductive options in the literature considered above or the Fregean abstraction view, our approach is not committed to the existence of determinate (or determinable) kinds of tropes. The talk about a determinate or determinable kind of tropes does not commit us to the existence of anything else than the tropes falling under that specific determinate (or determinable) quantity - that is, to a plurality of tropes standing in mutual basic internal relations of proportion and order. ${ }^{22}$ It remains to be shown that the truth of all propositions about certain kinds of tropes is determined by individual tropes. Similarly, the exact nature of the relevant truth-bearers is a matter for further study.

\section{Conclusion}

In the present article, we have put forth a new account of tropes of the same kind in terms of the basic internal relations of proportion and order. Tropes are in these basic internal relations due to them being the tropes they are: for example, particular determinate charges. As particular thin natures, tropes are basically internally related in certain ways. Considering proportions and order as basic internal relations entails the denial of their existence as any kind of relational entities. The proposed conception is superior to the standard account of tropes of the same kind: by means of the basic internal relations of proportion and order, we can specify the quantitative distances between tropes in a general and non-circular fashion, without assuming determinable properties or universals. The apparent advantage of the standard account - namely, the possibility of providing a conception of exact resemblance between quality tropes falling under

a determinate - turned out to be only apparent: typical quality tropes falling under a determinate, such as a determinate shade of red, can differ in some other respect. This frustrates

\footnotetext{
${ }^{22} \mathrm{We}$ are not committed to a one-to-many identity between determinate kinds of tropes and pluralities of tropes because we do not think that identity can be a one-to-many relation.
} 
the attempt to consider exact resemblances as internal relations that are not relativized in some definite respect.

In section 3 , we argued against the currently popular broadly reductive accounts of kinds of tropes and the Fregean abstraction account. They involve very problematic metaphysical assumptions, such as the postulation of sets or abstract individuals. Fisher's reductive view, which is a further development of Williams' Moderate Realism, avoids postulations beyond tropes. However, it has to make use of the false claim that mutually distinct tropes falling under the same determinate are identical, or else it would require a radical departure from the standard conception of identity as a single basic formal ontological relation.

Instead of subscribing to any of these problematic options, we suggested a new, parsimonious alternative: there are only tropes in different basic internal relations of proportion and order. Due to being basically internally related in certain ways, tropes themselves determine their falling under some specific determinate or determinable property. Hence, we do not postulate any other entities than just tropes, and do not identify shared properties with anything. What our theory does call for, however, is a meaning-theoretic account of the specific type of general talk - talk about shared properties and determinate/determinable kinds of tropes. In section 3.3, we indicated one possible avenue for developing such an account along the lines of the contemporary mainstream philosophy of language. A more detailed exposition of such an account must be left to future work; our brief sketch was intended to highlight that optimism in this regard is not ungrounded. ${ }^{23}$

\footnotetext{
${ }^{23}$ For helpful comments on earlier drafts of this article, we wish thank Guido Imaguire, Martin Pickup, Arto Repo, Gonzalo Rodriguez-Pereyra, Tuomas Tahko, Valtteri Viljanen, and the audiences at the University of Geneva, University of Durham, University of Oxford (TWIP seminar), University of Helsinki, University of Turku and Tampere University. We thank Finnish Cultural Foundation for funding this research.
} 


\section{References}

Armstrong, D.M. 1978, Universals and Scientific Realism, Cambridge: Cambridge University Press.

Armstrong, D.M. 1989, Universals - an Opinionated Introduction, Boulder: Westview Press.

Balashov, Y. 1999, 'Zero-Value Physical Quantities’, Synthese 119, pp. 253-286.

Betti, A. 2015, Against Facts, Cambridge Ma.: The MIT Press.

Bigelow, J. and Pargetter, R. 1990, Science and Necessity, Cambridge: Cambridge University Press.

Campbell, K.K. 1981, 'The Metaphysic of Abstract Particulars', Midwest Studies in Philosophy 6, pp. 477-488.

Campbell, K.K. 1990, Abstract Particulars, Oxford: Basil Blackwell.

Denkel, A. 1996, Object and Property, Cambridge: Cambridge University Press.

Ehring, D. 2011, Tropes: Properties, Objects, and Mental Causation, Oxford: Oxford University Press.

Ellis, B. 2001, Scientific Essentialism, Cambridge: Cambridge University Press.

Fisher, A.R.J. 2018, 'Instantiation in Trope Theory', American Philosophical Quarterly 55(2), pp. 153-164.

Giberman, D. 2014, 'Tropes in Space', Philosophical Studies 167 (2), pp. 453-472.

Giberman, D. 2016, 'Indiscernibility Does Not Distinguish Particularity', Thought: A Journal of Philosophy 5(4), pp. 249-256.

Hakkarainen, J. and Keinänen, M. 2017, 'The Ontological Form of Tropes - Refuting Douglas Ehring's Main Argument against Standard Trope Nominalism', Philosophia 45(2), 647-658.

Harré, R. 1970, The Principles of Scientific Thinking, London: Macmillan. 
Heil, J. 2012, Universe as We Find It, Oxford: Clarendon Press.

Johansson, I. 2000, 'Determinables as Universals', The Monist 1, pp. 101-121.

Keinänen, M. 2011, 'Tropes - the Basic Constituents of Powerful Particulars?', dialectica 65(3), pp. 419-450.

Keinänen, M. 2018, 'A Trope Theoretical Analysis of Relational Inherence', in Kuorikoski, J. and Toppinen, T., eds., Action, Value, and Metaphysics, Acta Philosophica Fennica 94, Helsinki: Societas Philosophica Fennica, pp. 161-189.

Keinänen, M., Keskinen, A. and Hakkarainen, J. 2017, 'Quantity Tropes and Internal Relations', Erkenntnis, published online.

Lewis, D.K. 1970, 'How to Define General Terms', Journal of Philosophy 67(13), pp. 427446.

Lewis, D.K. 1983, 'New Work for the Theory of Universals', Australasian Journal of Philosophy 61(4), pp. 343-377.

Lewis, D.K. 1986, On the Plurality of the Worlds, Oxford: Basil Blackwell.

Lowe, E.J. 2006, The Four-Category Ontology, Oxford: Oxford University Press.

Maudlin, T. 2007, Metaphysics within Physics, Oxford: Clarendon Press.

Maund, B. 2018, 'Color', in E.N. Zalta (ed.), Stanford Encyclopedia of Philosophy, Summer

2018 Edition, URL: https://plato.stanford.edu/archives/sum2018/entries/color/.

Maurin, A-S. 2002, If Tropes, Dordrecht: Kluwer Academic Publishers.

Morganti, M. 2009, ‘Tropes and Physics', Grazer Philosophische Studien 78, pp. 185-205.

Mulligan, K. 1998, 'Relations through Thick and Thin', Erkenntnis 48(2 \& 3), pp. 325-353.

Mumford, S. 2005, ‘The Ungrounded Argument', Synthese 149(3), pp. 471-489.

O’Conaill, D. \& Tahko, T. 2016, 'Minimal Truthmakers', Pacific Philosophical Quarterly 97(2), pp. 228-244.

Oliver, A. 1996, 'The Metaphysics of Properties', Mind 105(417), pp. 1-80. 
Papineau, D. 2016, 'Naturalism in Philosophy', in E.N. Zalta, ed., The Stanford Encyclopedia of Philosophy, Winter 2016 Edition, URL:

https://plato.stanford.edu/archives/win2016/entries/naturalism.

Rodriguez-Pereyra, G. 2002, Resemblance Nominalism - a Solution to the Problem of Universals, Oxford: Oxford University Press.

Rodriguez-Pereyra, G. 2017, 'Indiscernible Universals', Inquiry: An Interdisciplinary Journal of Philosophy 60(6), pp. 604-624.

Rosen, G. 2014, 'Abstract Objects', in E.N. Zalta, ed., The Stanford Encyclopedia of Philosophy, Fall 2014 edition, URL:

http://plato.stanford.edu/archives/fall2014/entries/abstract-objects.

Simons, P.M. 1987, Parts - a Study in Formal Ontology, Oxford: Clarendon Press.

Simons, P.M 1994, 'Particulars in Particular Clothing - Three Trope Theories of Substance', Philosophy and Phenomenological Research 54(3), pp. 553-575.

Simons, P.M. 1998, 'Farewell to Substance - a Differentiated Leave-Taking', Ratio 11, pp. $235-251$.

Simons, P.M. 2003, 'Tropes, Relational', Conceptus 35, pp. 53-73.

Simons, P.M. 2008, 'The Thread of Persistence', in Kanzian, C., ed., Persistence, Frankfurt: Ontos Verlag, pp. 165-184.

Williams, D.C. 1953a, 'On the Elements of Being I', Review of Metaphysics 7, pp. 3-18.

Williams, D.C. 1953b, 'On the Elements of Being II', Review of Metaphysics 7, pp. 171-192. Williams, D.C. 1986, 'Universals and Existents', Australasian Journal of Philosophy 64(1), pp. 1-14. 